\title{
Un double monstrueux : la culture criminelle en Russie post-soviétique (partie 2)
}

\section{Anton Oleinik}

\section{(2) OpenEdition}

1 Journals

\section{Édition électronique}

URL : http://journals.openedition.org/conflits/575

DOI : $10.4000 /$ conflits. 575

ISSN : $1777-5345$

Éditeur :

CCLS - Centre d'études sur les conflits lilberté et sécurité, L'Harmattan

Édition imprimée

Date de publication : 1 juin 2001

ISBN : 2-7475-1117-0

ISSN : 1157-996X

\section{Référence électronique}

Anton Oleinik, « Un double monstrueux : la culture criminelle en Russie post-soviétique (partie 2)», Cultures \& Conflits [En ligne], 42 I été 2001, mis en ligne le , consulté le 30 mars 2021. URL : http:// journals.openedition.org/conflits/575; DOI : https://doi.org/10.4000/conflits.575

Ce document a été généré automatiquement le 30 mars 2021.

Creative Commons License 


\title{
Un double monstrueux : la culture criminelle en Russie post-soviétique (partie 2)
}

\author{
Anton Oleinik
}

Dans la société carcérale, tout comme dans la société de type soviétique, les relations personnalisées jouent un rôle essentiel dans la vie sociale et ne se réduisent pas complètement à une logique traditionnelle et hiérarchique. Le terme de réseau, utilisé plus haut pour décrire les relations inter-entreprises, illustre l'importance des relations personnalisées mises au service d'un projet. L'institution familiale occupe une place centrale dans la construction sociale des deux sociétés. La famille ne peut cependant être réduite à la logique des relations traditionnelles. Dans le cas de la société carcérale, les détenus choisissent librement leur kentovka, ils peuvent en changer volontairement ( $46 \%$ des personnes interrogées ont changé de «famille » une ou plusieurs fois). Dans le cas de la société de type soviétique, la famille russe échappe au modèle traditionnel pur. Selon une enquête ${ }^{1}, 76 \%$ des familles interrogées sur leurs relations intrafamiliales considèrent que toutes les décisions importantes sont prises de façon consensuelle, $58 \%$ sont persuadées qu'aujourd'hui «le mari et la femme doivent partager d'une façon égale les tâches d'éducation des enfants, de ménage, de maintien du bien-être ", 71\% pensent que l'existence chez les conjoints de cercles d'amis et de connaissances différenciées aide la famille, et $77 \%$ que la famille se fonde sur des droits et obligations réciproques. Les institutions auxquelles les Russes font confiance

«Qu'est-ce qui vous inspire la confiance ?» Personnes interrogées au sein de la société russe $^{2}$ Détenus interrogés La famille $71 \%$ 66\% Les amis 45 Données absentes L'église 24 19 La science 174 Les collègues / les codétenus 812 Le peuple 76

Pour la plupart des Russes, la confiance constitue l'un des socles de la vie familiale. 57\% des personnes interrogées ${ }^{3}$ considèrent la confiance comme le deuxième régulateur des relations entre les conjoints (la capacité de comprendre l'autre figure en premier), 54\% lui attribuent le même rôle dans les relations entre parents et enfants (ici, c'est l'amour qui arrive en tête de la liste de réponses avec 58\%). En réponse à la question plus 
générale "Qu'est-ce qui régule les relations dans votre famille ?», 86\% choisissent la confiance (avant l'amour, les obligations morales, etc.). L'importance significative de la confiance dans les relations intra-familiales s'oppose à la méfiance presque totale qui apparaît aussitôt qu'on traverse la frontière de la famille et des relations personnalisées. Seul $32 \%$ des personnes que nous avons interrogées pensent qu'on "peut faire confiance aux gens ». Le graphique ci-dessous montre comment évolue la confiance envers les autres dans la société russe des années 19904. A titre de comparaison, le niveau minimal de la confiance dans les sociétés occidentales, mesuré de la même manière, ne dépasse pas le seuil de $60-70 \%^{5}$.

La frontière entre les siens et les autres peut être tracée de différentes façons. L'appartenance à la famille et la connaissance personnelle sont bien les conditions nécessaires pour être admis parmi les siens. Comme le montre une enquête récente, les siens sont avant tout les membres de sa famille (89\%), ses amis (84\%), ceux qui partagent une vision commune du monde (76\%), ceux qui sont de la même génération $(72 \%)^{6}$. Par conséquent, dans la société soviétique et post-soviétique, tout comme en prison, la confiance envers les siens apparait comme le revers d'une méfiance presque totale envers les autres, ceux qu'on ne connait pas personnellement. Le dernier point de comparaison porte sur les moyens de gérer la violence. La violence au quotidien est très répandue dans la société post-soviétique, bien qu'elle ne prenne pas nécessairement une forme brutale. Les bousculades dans la rue et dans les transports en commun, les insultes verbales qu'on appelle «incivilités» représentent des éléments importants du décor post-soviétique. Plus d'un tiers des Soviétiques se souviennent par exemple des « insultes verbales » qu'ils ont récemment essuyées?. Une part importante des « incivilités » reste cachée : soit les gens ne les perçoivent pas en tant que telles, soit ils en tiennent compte sans les rendre publiques. D'une part, "l'individu victime d'un acte de violence ressentira les conséquences de cet acte suivant l'inscription de l'acte dans une structure de perception qui lui préexistera ${ }^{8}$. Les incivilités passent souvent inaperçues parce qu'elles deviennent de plus en plus des normes de comportement. «La violence ici, c'est avant tout les bagarres, parce qu'elles sautent aux yeux» (\#13). D'autre part, les gens ne rapportent pas fréquemment les actes de violence subis même lorsqu'ils figurent dans le Code Pénal. Selon une étude sociologique sur ce sujet, le niveau de criminalité latente atteindrait en Russie 70\%, notamment parce que $40 \%$ des victimes d'actes violents ne les rapporteraient pas à la police'. La discussion sur les raisons de cette décision exigerait un développement séparé. Nous nous contentons de mentionner la forte présence de la violence, rapportée ou non, dans la vie quotidienne. La gestion de la violence soviétique implique la recherche d'un bouc émissaire qui permet de la canaliser vers une cible bien définie. La recherche d'une victime de rechange s'effectue à l'extérieur et à l'intérieur de la société soviétique. Il a été montré que la guerre figurait au second rang des peurs de l'homme soviétique ordinaire (elle est mentionnée par $47 \%$ des personnes interrogées), juste après la maladie d'un proche (mentionnée par $59 \%)^{10}$. La fin de la guerre froide n'a pas réduit cette peur d'un ennemi étranger, puisque $24 \%$ des Russes post-soviétiques considèrent qu'une agression externe est toujours probable ${ }^{11}$. Les ennemis intérieurs ont revêtu divers aspects au cours de l'histoire soviétique: koulaks, trotskistes, Allemands nés en Union Soviétique, vrediteli («ceux sur lesquels la bureaucratie soviétique rejetait la responsabilité des résultats de la politique économique » ${ }^{12}$ ) et autres durant les années 1930-1950. Au cours des années 1960-1980, les touneïadtsy, c'est-à-dire ceux qui refusaient de travailler dans une entreprise d'Etat, représentaient 
toujours une figure emblématique de l'ennemi intérieur ${ }^{13}$. Durant les années 1990, ce sont les directeurs d'entreprises, les banquiers, les communistes, les fonctionnaires d'Etat, les criminels qui ont tour à tour ou simultanément incarné la figure de l'ennemi dans la conscience populaire ${ }^{14}$. Les deux guerres de Tchétchénie peuvent être considérées comme une tentative des détenteurs du pouvoir en Russie de trouver une victime idéale : "Il faut que la victime ne soit ni trop ni pas assez étrangère à cette même communauté » ${ }^{15}$. Les criminels et la criminalité organisée figurent en tête de ce répertoire des nouveaux «ennemis ». $61 \%$ des personnes interrogées les considèrent comme l'un des trois groupes, avec les banquiers et les fonctionnaires, qui ont le plus bénéficié des réformes économiques des années $1990^{16}$. On reproche surtout à la criminalité organisée un rôle négatif dans le domaine économique. Le racket, les prêts à usure, la «surveillance » de tous les secteurs d'activité sont devenus indissociables de l'image du marché russe. Comme en prison, la surveillance signifie l'omniprésence et la capacité de contrôle, c'est-à-dire l'autorité. «Etiez-vous au courant des flux de capitaux dans l'entreprise? - Non. - Donc, vous ne la contrôliez que partiellement ? - Non, je la contrôlais en totalité » (\#5). « Vous parlez toujours de 'votre' magasin [magasin sous la 'surveillance' du criminel] bien qu'il appartienne à quelqu'un d'autre. Qu'est-ce que vous entendez par 'votre' magasin? - C'était dans les faits mon magasin. Parce qu'il était sous mon autorité » (\#23). Venons-en à notre troisième hypothèse, relative au lien entre le monde carcéral et le marché post-soviétique. Selon les estimations, le secteur informel de l'économie russe a représenté à la fin des années 1990 de 25 à $40 \%$ du Produit National Brut ${ }^{17}$. Par économie informelle, nous entendons l'ensemble des activités économiques qui se réalisent sans référence à la loi et aux dispositifs légaux. Le marché criminel, qui sous-entend la présence d'une tutelle criminelle, et les autres secteurs de l'économie informelle doivent être distingués. La tutelle criminelle est assurée par les smotriachtchie, dont l'influence ne se limite pas à la société carcérale et qui remplissent d'une manière générale quatre fonctions auprès des acteurs du marché

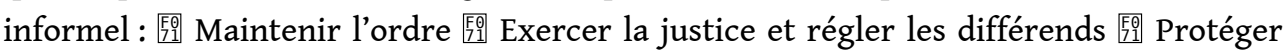
contre la criminalité non organisée 囲 Prêter à usure Tout comme dans le monde carcéral, les divers secteurs du marché informel possèdent des "constitutions " informelles qui gouvernent les activités quotidiennes. Malgré leur caractère non écrit, ces normes sont connues de tous les acteurs impliqués dans le marché informel. C'est par exemple le cas du code de la morale commerciale des années 1960 : le vol est toléré, l'honnêteté n'est réservée qu'aux Don Quichotte, l'homme est un loup pour l'homme, la conscience doit être élastique, un innocent est un voleur qui n'a pas été attrapé, tout repenti deviendra un paria, etc. ${ }^{18}$ L'évidente dualité des normes ne doit pas surprendre : à cette époque, il était souvent question de "mafia commerciale ", une personne extérieure au réseau de connaissances dans le secteur commercial ne pouvait jamais trouver un poste dans un magasin plus ou moins prestigieux, qui favorisait automatiquement l'accès à des biens déficitaires. Bien que les normes illicites se développaient et s'appliquaient spontanément, l'intervention des smotriachtchie pour maintenir l'ordre illicite était parfois nécessaire. Ils préservaient du bespredel, c'est-àdire qu'ils veillaient au respect des normes informelles. «La fonction du smotriachtchii est de garantir le respect des règles. S'il était absent, il y aurait le bespredel. - Vous parlez du business ? - Du business tout comme de la société criminelle. Il y aurait des tirs à la Kalachnikov partout. Si tu t'adresses aux smotriachtchie, leur justice peut te protéger contre le bespredel. C'est la seule chose qu'ils peuvent faire, mais tu seras obligé de les payer. De même si ta protection est assurée par les organismes officiels. 
Chez ces derniers, les tarifs sont plus élevés. -Un recours en justice peut-il être envisagé ? - Nullement. - Dans aucun des deux cas ? - Il n'y a pas de différence. Quand j'étais libre, mes gardes de corps provenaient des institutions répressives, mais il m'arrivait en même temps de m'adresser aux smotriachtchie» (\#25). Depuis la fin des années 1980 , le «maintien de l'ordre» figure au premier plan de l'activité des smotriachtchie. Le rôle de médiateur dans les conflits entre les agents de l'économie informelle s'observe depuis la fin des années 1960. Les smotriachtchie interviennent non seulement dans les conflits, mais jouent également parfois un rôle de médiateur strictement commercial ${ }^{19}$. La médiation commerciale rappelle la production et la vente de la confiance par la Mafia sicilienne. Lorsque les parties d'un contrat illicite ne se connaissent pas mais sont en relation avec un même smotriachtchii, la médiation de ce dernier, dont la réputation personnelle est connue des deux parties, devient inévitable. La question des honoraires du médiateur reste ouverte. Selon certains auteurs, « [les smotriachtchie] ne demandent pas de rémunération pour leur arbitrage. Ils attendent un service en retour de la part des solliciteurs, si besoin est $"{ }^{20}$. Cependant, pour certains détenus interrogés, le recours au patronage des smotriachtchie coûterait assez cher. «Comment les commerçants paient-ils le service? Je ne suis pas au courant de la situation actuelle car je suis emprisonné depuis déjà longtemps, mais avant il y avait un pourcentage fixé par rapport au chiffre d'affaires qu'on ne pouvait ni augmenter, ni diminuer. En somme, il existait avant un pourcentage unique»(\#4). Les sources policières confirment l'existence d'un pourcentage fixe, semblable au pizzo pratiqué par la Mafia sicilienne ${ }^{21}$ : une réunion de « voleurs dans la loi » (skhodka) à Kislovodsk (Caucase) en 1979 aurait déterminé un tarif représentant 10\% des bénéfices nets. Ce prix aurait doublé vers la fin des années 1980 (20-25\% des bénéfices nets) ${ }^{22}$. La rémunération inclut également le prix de certains autres services rendus par les smotriachtchie, en particulier celui de la protection contre la criminalité non organisée, c'est-à-dire les petits racketteurs, les pilleurs, les escrocs, les voyous. « Votre ami, qui est commerçant (coopérateur), vous a-t-il demandé quelque chose de précis ? Il m'a proposé de travailler avec lui. - A quel titre ? - Je lui ai moi-même posé cette question. Il expliquait qu'il fallait le suivre partout, l'assurer de ma présence. Quand j'ai demandé des précisions, il est apparu qu'il cherchait un garde du corps. Il ne me l'a pas dit directement, mais il me l'a fait comprendre »(\#24) ${ }^{23}$. Paradoxalement, la protection criminelle réduit le taux de criminalité. Il existe même des preuves statistiques de l'existence d'une corrélation négative entre le taux de criminalité et le développement du marché informel. Au niveau régional, plus les transactions informelles sont importantes et mieux organisées, moins il y a de la criminalité ${ }^{24}$. Ce paradoxe peut expliquer la réputation actuelle, largement diffusée en Russie, de Saint-Petersbourg comme capitale criminelle et ville de bespredel ${ }^{25}$. Certains de nos interlocuteurs ont souligné le fait que cette ville était l'un des rares endroits sans smotriachtchie. "Le bespredel vient de là. Il existe des groupes criminels qui ne reconnaissent pas les smotriachtchie. Surtout à Petersbourg. Je n'ai pas été là-bas, mais je le sais très bien. Dans la prison des Kresty ${ }^{26}$, personne n'est unanimement reconnu. Pour eux, il n'y a aucune différence entre un «voleur dans la loi » et un autre» $(\# 18)^{27}$. Le volume des fonds contrôlés par les smotriachtchie est significatif : aux impôts illicites payés par les acteurs de l'économie informelle s'ajoutent les contributions volontaires des criminels dits "fiers", c'est-à-dire défenseurs des valeurs de la culture carcérale. Ceux-ci transférent dans des fonds communs (obchtchak) une partie de leurs revenus, principalement issus des vols et cambriolages effectués aux dépens des individus non 
protégés par les smotriachtchie, "Quand j'habitais à Simferopol [en Crimée], je connaissais celui qui contrôlait l'obchtchak de la ville. Lorsqu'on volait quelque chose, je décidais ou non d'y verser une part de butin. Personne ne me forçait à le faire. Personne n'avait le droit de me dire : 'Toi, tu es obligé d'y contribuer'. Nous, on était une bande de quatre hommes. Une fois on a volé trois-quatre mille roubles. On a alors décidé qu'on verserait à l'obchtchak deux à trois cents roubles. Je connaissais alors Moulia, aujourd'hui décédé, qui contrôlait à l'époque l'obchtchak. On est allé chez lui et on lui a dit 'Salut, Moulia, tiens, de notre part. C'est notre contribution à la chose commune'. Pour les fonds prélevés chez les commerçants, c'est pareil. Les bandes qui imposent le paiement d'une somme aux commerçants doivent en transférer une partie à l'obchtchak. Une partie est conservée, le reste va à l'obchtchak. Pareil pour les sommes reçues au titre d'une protection. Habituellement, c'est un voleur dans la loi qui contrôle l'obchtchak» (\#18) ${ }^{28}$. L'importance des fonds collectés par les smotriachtchie permet non seulement d'aider les criminels arrêtés par la police, mais aussi d'effectuer des prêts aux agents du marché informel. Sur le marché criminel, le crédit se transforme souvent en moyen de contrôler l'emprunteur. La logique de l'assujettissement à long terme prévaut par rapport à celle de la maximisation des profits à court terme. "Perceviez-vous des intérêts? - Je ne percevais pas d'intérêts. Par principe. - Quel principe? En quoi consistait votre intérêt? - J'attendais des services en retour. L'emprunteur pouvait me rendre un service dans l'avenir. - Que pouviez-vous demander comme service en retour? - Qu'il signe un document. Un permis de construire, une autorisation de vente... » (\#5) ${ }^{29}$. Il n'est pas exclu que cet échange prenne une forme violente. Les criminels essayent parfois d'endetter artificiellement leurs clients afin de pouvoir ensuite les manipuler, surtout si ceux-ci ne font pas vraiment partie des " leurs ». "Pourquoi nous adressions-nous aux criminels? $\mathrm{Au}$ début, on ne pensait pas aux problèmes éventuels. Il était plus facile d'emprunter à ces types. "Enfin, tu as décidé de nous parler, tu es des nôtres »- m'ont-ils dit. La première fois que je leur ai emprunté de l'argent, j'ai rapidement ressenti leur puissance et mon impuissance. On avait un excavateur, avec lequel on extrayait du sable pour le mettre en vente. [Les criminels] ont placé une grenade sous l'excavateur et nous avons perdu trois jours pour le réparer. Ils te prêtent de l'argent et trouvent aussitôt des moyens d'assécher tes sources des revenus. Ils font ça pour qu'on ne puisse pas rembourser la dette. Ils exigent alors des intérêts exorbitants et commencent à te manipuler comme ils veulent " (\#6). Les entrepreneurs russes sont très sceptiques quant à l'appui sur la criminalité organisée dans leurs affaires. D'un côté, ils recherchent parfois cet appui après avoir éprouvé l'inefficacité du système judiciaire dans la résolution des conflits commerciaux. "[Soumettre un contentieux] dure longtemps. Dans le meilleur des cas, cette procédure peut vous aider à récupérer $30 \%$ de vos pertes et de vos dépenses judiciaires » $\left(\# 3^{*}\right)^{30}$. «Le jugement officiel vous permet de ne pas rembourser les sommes indues. S'il s'agit d'un différend, vous attendez le dédommagement. Hélas, vous obtenez le jugement, mais pas le dédommagement. Le système est loin d'être parfait, il existe beaucoup de voies pour le contourner » $\left(\# 20^{*}\right)$. «On soumet un contentieux. On attend un an et on demande le jugement. On l'obtient et on le montre au débiteur. On lui demande de rembourser. Après on s'assoit et on attend. Rien ne se produit. On s'adresse à la police : toujours rien. C'est seulement après qu'on décide de s'adresser aux criminels et que les choses commencent à bouger. Parfois ça aide, parfois ça rend les choses encore plus graves » (\#9*). D'un autre côté, le mode criminel de résolution des conflits commerciaux, bien qu'il soit parfois plus 
efficace, coûte cher et crée une dépendance vis-à-vis de la criminalité organisée. « Demander l'aide de la criminalité organisée pour récupérer de l'argent prêté est une chose ingrate car, dans le meilleur des cas, on n'obtient que $50 \%$ de la somme prévue. De toute façon ça perturbe votre travail, ça prend beaucoup de temps, ça vous met dans un état de grande nervosité. Il arrive souvent que la police poursuive les criminels avec qui vous êtes en affaire, - et même si la police ne vous poursuit pas, le règlement de ces problèmes bloque les activités commerciales durant une certaine période » $\left(\# 2^{*}\right)$. Du point de vue des entrepreneurs, aucune priorité absolue n'est donc accordée ni au système légal, ni au système criminel. Cette constatation peut sembler paradoxale tant qu'on n'observe pas en détail les avantages et les inconvénients de chaque système de justice. En règle générale, on se soumet au principe « La force appuie le droit » dans les deux cas. «La partie qui bénéficie du soutien le plus puissant a toujours raison » (\#16*). " Il existe un principe selon lequel le 'toit' (krycha) ${ }^{31}$ soutient la justice» ${ }^{32}$. " La résolution du conflit dépend de votre adversaire. Si votre adversaire est plus fort que vous, - il faut dire 'au revoir' à votre argent et il faut même se réjouir de rester en vie. Si votre adversaire est moins fort que vous - on s'adresse aux criminels qu'on connait et on résout le problème » $\left(\# 5^{*}\right)$. « [Les organismes officiels et la criminalité organisée] s'entendent bien. Ils trouvent très vite un terrain d'entente» (\#25). Dans un tel contexte, le recours aux organismes d'Etat pour appuyer une partie du conflit commercial au détriment de l'autre ne doit pas surprendre. Comme nous l'avons déjà remarqué, il n'y pas de différence qualitative entre le 'toit' criminel et le 'toit' officiel. «Le 'toit'? Ha! Ha! Ce terme est susceptible d'être interprété de plusieurs manières. Nous sommes les clients des organismes officiels » $\left(\# 16^{*}\right)$. Il y a tout simplement plus de chances que le 'toit' officiel soit le plus puissant. « En cas de conflit, on peut arranger les choses afin que l'entrepreneur qui ne vous plaît pas cesse bientôt ses activités. L'Inspection fiscale lui rendra 'par hasard' une visite, ou le Contrôle sanitaire et épidémique s'intéressera à sa firme... Une fois ces gens sur place, il est impossible de s'en débarrasser. Ils te connectent une pompe et commencent à pomper l'argent. Sans fin » (\#12*). Ainsi l'Etat joue-t-il un rôle selon les mêmes règles observées à propos de la société carcérale. Les réponses à la question «Qu'entendez-vous par 'Mafia'?» illustrent la diffusion des représentations d'un Etat respectueux des normes mafieuses. Il s'agit de l'économie parallèle pour $43 \%$ des personnes interrogées, des bureaucrates dans les ministères et les administrations pour $36 \%$, de l'appareil du parti-Etat pour $34 \%$ - des criminels pour $25 \%^{33}$. Le scepticisme des entrepreneurs russes par rapport à l'Etat prouve la nature disjointe de son autorité. Le faible niveau de confiance des Russes envers l'Etat prolonge ce constat. Cette méfiance, déjà significative avant la crise du mois d'août 1998, a pris par la suite des formes extrêmes. Seul 9\% des personnes que nous avons interrogées en 1998 pensaient qu'on pouvait faire confiance à l'Etat. Leur nombre a diminué par trois $(3,4 \%)$ en 1999. Le degré de confiance envers l'administration dans les prisons $(25,1 \%)$ dépasse largement le degré général de confiance envers l'Etat !: «Toutes les réformes précédentes en Union Soviétique ont été considérées par les citoyens comme un 'grand mensonge'. La seule voie qui permettra de sortir de cette impasse consiste à engager l'Etat de façon obligatoire et crédible dans la politique de réformes " ${ }^{34}$. La situation actuelle nous persuade malheureusement que les post-Soviétiques continuent à ne voir dans les actions de l'Etat qu'un grand mensonge. L'Etat reste ignorant des intérêts de ses citoyens et seuls ses besoins fiscaux le rendent dépendant d'eux. Si l'Etat et la criminalité organisée se disputent le même champ d'activité et utilisent les mêmes repères normatifs, ils 
deviennent alors adversaires. Bien qu'un parallèle direct avec la situation sicilienne des années 1930 (sous l'autorité du préfet fasciste Cesare Mori) soit peu justifié, cette dernière fournit un exemple de rivalité entre une Mafia et un Etat mafieux: «Pour combattre la Mafia, l'Etat s'était transformé en une autre Mafia $"{ }^{35}$. Simultanément, la lutte contre la criminalité organisée s'inscrit dans une logique de recherche d'une victime de rechange, mentionnée plus haut. A la différence des «terroristes" tchétchènes, la criminalité organisée est présente partout dans la Russie actuelle. En outre, et on a essayé de le démontrer, la criminalité organisée n'est pas étrangère à la société et au marché post-soviétiques, qui se construisent autour des mêmes normes et valeurs. La criminalité organisée, en tant que victime de rechange, offre un avantage majeur, celui de pouvoir être sacrifiée. Par conséquent, la criminalité organisée est devenu un double monstrueux parfait, que l'Etat peut exploiter pour cibler la violence tout en étant assuré du soutien de la population. Ce double monstrueux de la société post-soviétique n'est ni complètement dedans, ni complètement dehors : il réside dans un lieu de tension entre une volonté d'expulsion totale et un désir d'incarnation également total.

Annexes A. Liste de personnes interrogées

\# Age, catégorie ( $\mathrm{B}=$ blatnoï, $\mathrm{K}$ = koziol, $\mathrm{M}$ = moujik) Caractéristiques complémentaires 1 Andreï, 35-40 ans, B Smotriachtchii d'un régiment, deuxième détention (1989 à 2004) pour racket, originaire de Yaroslavl 1a Alexeï, 20-25 ans, B Bras droit d'Andreï, dernière détention de 1994 à 2000 pour racket, originaire de Yaroslavl 2 M Originaire de Gorki 3 Viktor, plus de 35 ans En détention dans une prison pour jeunes délinquants de 1984 à 2002, a commis un meurtre en prison, une tentative d'évasion 3a Andreï Yourevitch, 30-35 ans Directeur adjoint de la Maison centrale, responsable de l'éducation 4 Valeri, 35-40 ans, B Smotriachtchii d'un régiment, en détention depuis 1992, originaire de Klin (région de Moscou) où il était smotriachtchii, déjà détenu dans une prison pour jeunes délinquants 5 25-30 ans, B Chef d'un gang de racketteurs à Lioubertsy (banlieue de Moscou), deuxième détention, cherche à obtenir un statut d'autorité informelle 6 Plus de 35 ans

Escroc, originaire de Crimée (Ukraine), première détention 7 « Mironytch ", plus de 50 ans, M Arestant ${ }^{36}$, en détention de 1995 à 2005, se référant aux valeurs traditionnelles, a déjà été plusieurs fois incarcéré 8 20-25 ans D'origine paysanne, première détention, pour racket 9 German, 27 ans, K Originaire de Toula, escroc, responsable (zavkhoz) d'un détachement regroupant les criminels les plus endurcis, première détention de 1994 à 2000, smotriachtchii d'une cellule (8 personnes) dans une Maison d'arrêt 10 Plus de 30 ans, K Escroc, originaire de Moldavie, en détention depuis 1997, responsable d'un détachement (zavkhoz) 11 Plus de 40 ans, K Responsable d'un détachement (zavkhoz) regroupant des kozly, ex-officier de la police routière, première détention, smotriachtchii d'une cellule dans une Maison d'arrêt 12 Plus de 50 ans, M Copain du $\mathrm{n}^{\circ} 7$, arestant, plusieurs fois en détention 13 Vladimir, plus de 50 ans, M Détenu dans les années 1970, actuellement en détention (1993-2003), auteur d'un meurtre commis en prison, originaire de Toula, croyant 14 Plus de 40 ans, M Détenu dans les années 198015 25 ans Ancien officier des douanes, moscovite, en détention pour la première fois (1998-2004) 16 Vladimir, 40+ ans, M En détention dans les années 1970, auteur du meurtre de son frère, copain du $\mathrm{n}^{\circ} 7$

1750 ans, M Originaire de la région de Kostroma, en détention dans une prison pour jeunes délinquants dans les années 19601850 ans, M Régulièrement en détention 
depuis 1967 (7 détentions, 20 ans au total, la dernière fois de 1997 à 1999), voleur, originaire de Crimée, ukrainien, arestant 19 Plus de 20 ans, M Originaire de la région de Toula, en détention de 1997 à 2004 pour crime économique, niveau de première année universitaire 20 Sergeï, moins de 18 ans, M En détention de 1998 à 2001 pour infraction à la législation sur les stupéfiants 21 Nikolaï, 51 ans, $\mathrm{M}$ En détention à plusieurs reprises depuis les années 1970, aujourd'hui en détention depuis 1997, originaire de SaintPeterbourg, arestant 22 Nikolaï, plus de 50 ans, M En détention à plusieurs reprises depuis 1968, originaire de Saint-Peterbourg, aujourd'hui en détention jusqu'en 2006 pour vol avec violence 23 Plus de 30 ans, B Racketteur, originaire de Tver, détention en QHS 2442 ans, Youri, B Originaire de la région de Novgorod, plusieurs fois en détention (y compris dans une prison pour jeunes délinquants) depuis les années 1970, dernière détention de 1991 à 1999, auteur d'un meurtre, en QHS depuis deux ans, smotriachtchii d'un détachement 25 Plus de 30 ans, B Originaire de Bakou, commerçant à Moscou, en détention de 1995 à 2001 pour escroquerie, en internat de 3 à 14 ans, smotriachtchii d'une cellule à la Maison d'arrêt 26 Vladimir, plus de 40 ans Responsable de l'éducation 27 Sergeï, plus de 30 ans, B Smotriachtchii des jeux de cartes, voleur

B. Questionnaire

1. De votre point de vue, peut-on généralement faire confiance aux gens? 媦 Oui 期 Non

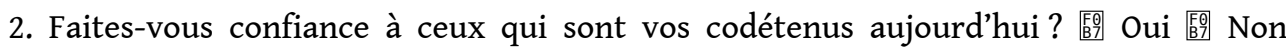
(mettre une croix dans la case correspondante) 3. Quels sont vos amis aujourd'hui ? 4.

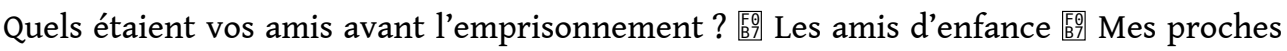
匑 Les camarades de classe 盛 Mes camarades de cellule aujourd'hui

3. Avez-vous reçu des visites de vos proches au cours du dernier mois? 期 Oui 䒜 Non 4. Recevez-vous souvent des lettres de vos proches? 區 Oui, chaque semaine 䁒 Oui, deux

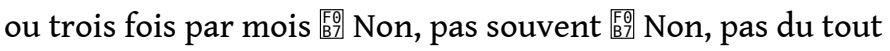

5. Allez-vous maintenir des relations avec les codétenus après votre libération? 費 Oui 范 Non 閶 Je ne sais pas 6 . Comment décrivez-vous votre situation psychologique

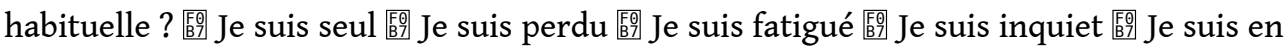
colère 覧 Je suis patient 婜 Je tente de changer ma vie (mettre une croix dans la case correspondante) 7. Qui vous sert d'appui dans une situation difficile? 8. Qui vous a aidé



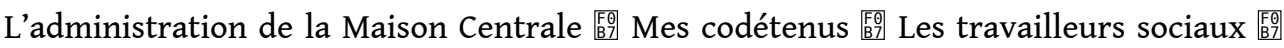
L'Etat

7. Travaillez-vous durant la détention? 䜿 Oui 䜿 Non 8. Quelles sont les qualités que

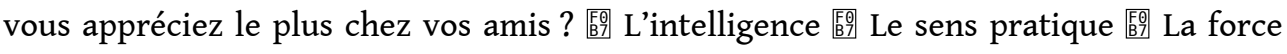



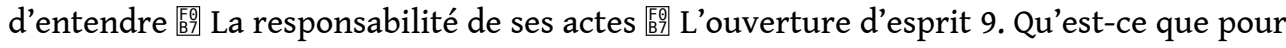

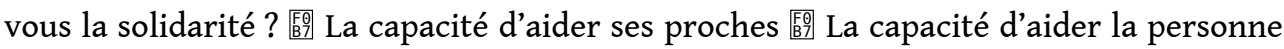
qui se trouve dans la même situation que soi 媦 L'espoir d'un comportement réciproque 㽚 Le soutien apporté aux revendications justes 10. Etes-vous prêt à participer à une

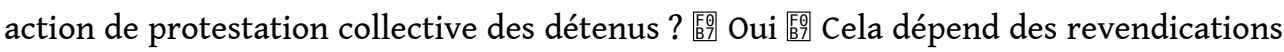
區 Cela dépend des gens qui y participeront 圆 Non 11. Quel mode de résolution des



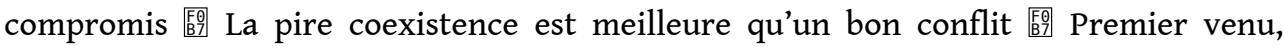
premier servi 鍳 Les meilleures solutions sont trouvées habituellement par les personnes sages 
12. Quelle est l'intervention la plus efficace en cas de conflit dans le milieu carcéral (entre détenus)? 撂 L'absence d'une intervention externe par rapport aux parties du

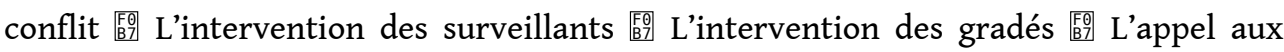
autorités judiciaires 㯺 L'intervention d'un détenu que les parties du conflit respectent

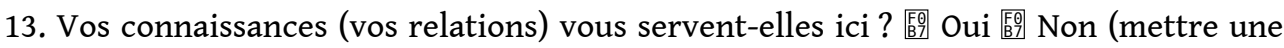
croix dans la case correspondante) 14. Qu'est-ce que vous feriez si vos droits étaient violés par un surveillant? 15. Qu'est-ce que vous feriez si vos droits étaient violés par

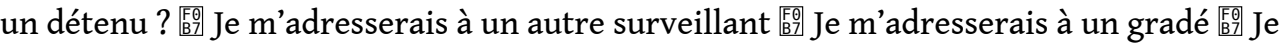
m'adresserais à mon avocat 䔽 Je m'adresserais à une association de défense des droits des détenus 14. La violation de quel type de règles jugez-vous la plus dangereuse? 睓 Le

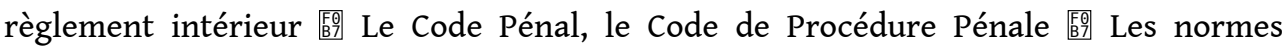
informelles qui servent d'appui dans les relations avec des surveillants 䀝 Les normes informelles qui servent d'appui dans les relations avec les autres détenus 15. Quelles sont les qualités que vous pourriez respecter chez un détenu? 臨 L'intelligence 阔 Le

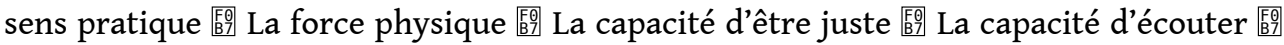

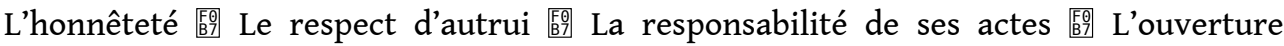
d'esprit 16. Pourquoi remplissez-vous vos obligations par rapport à autrui ? 㕆 Je respecte autrui 區 J'espère prolonger les relations avec cette personne 瞖 J'essaie de maintenir ma réputation 菏 Je sais qu'un comportement opportuniste sera tôt ou tard puni 17. Maintiendriez-vous des relations avec une personne désagréable mais utile? 區

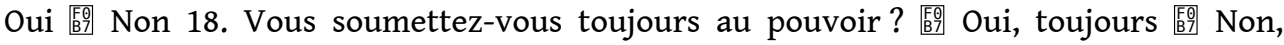
seulement si la défense de mes intérêts coûte cher 嘔 Non, seulement si l'utilisation du pouvoir ne contredit pas mes intérêts 堅 Non, jamais 19. Qu'est-ce qui est le plus

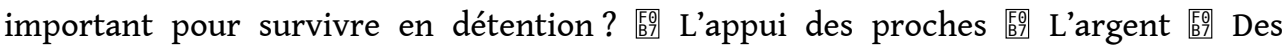

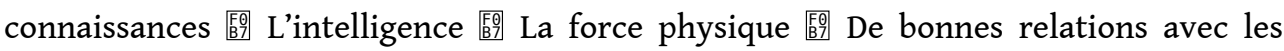
surveillants 臨 De bonnes relations avec d'autres détenus

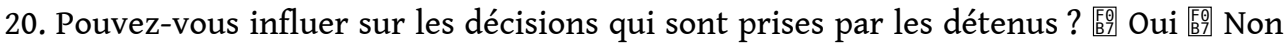

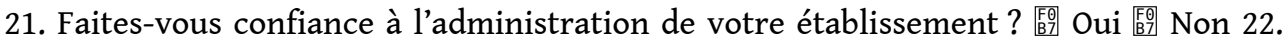

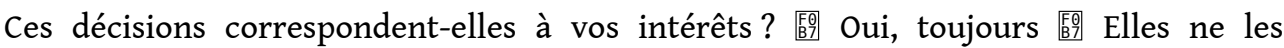

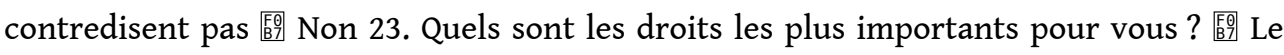

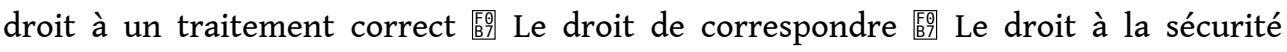

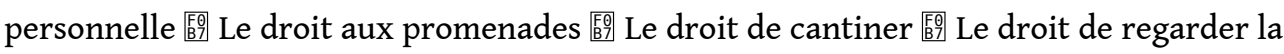

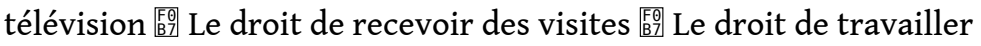

24. Pourriez-vous vous adresser directement, si c'était nécessaire, 盛 à l'administration

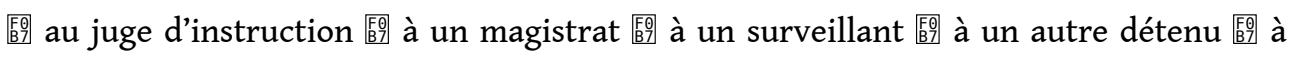
une association ayant la vocation de défendre les droits des détenus 谌 à un avocat

25. Considérez-vous que vous pouvez réaliser vos droits (mettre une croix)

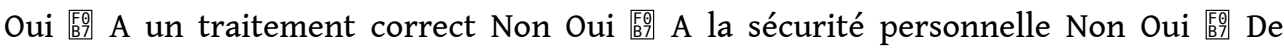

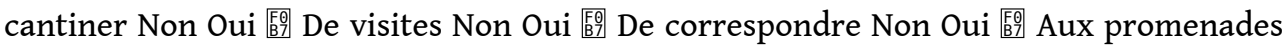
Non Oui 圆 De regarder la télévision Non Oui 圆 De travailler Non

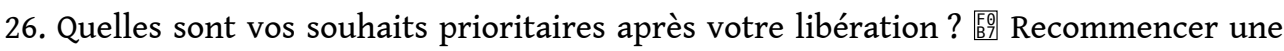

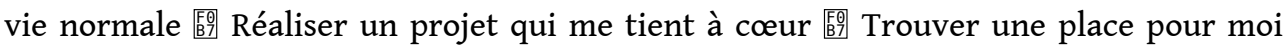
dans la société 27 . Quels sont les moyens pour les atteindre? 婜 Le respect de la Loi 㹂

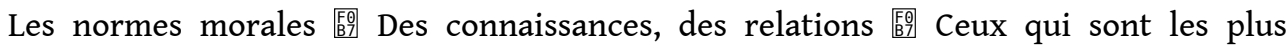



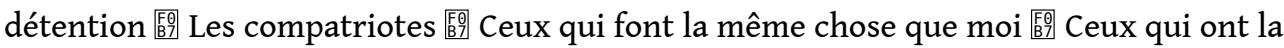
même vision du monde que moi [⿰⿷匚⿳丨コ丨⿱⿰㇒一乂 $\mathrm{J}$ Je ne distingue pas les gens de cette façon 


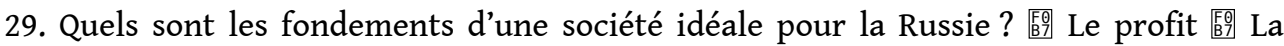

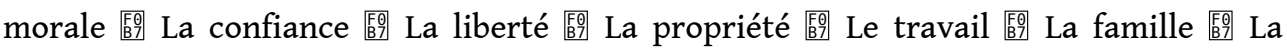

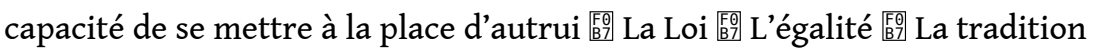

30. Vous sentez-vous responsable de / des: 范 Vos actes Oui Non 范 Actes des vôtres Oui

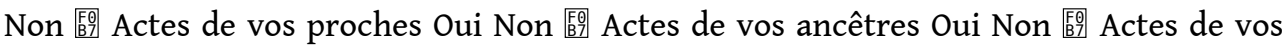

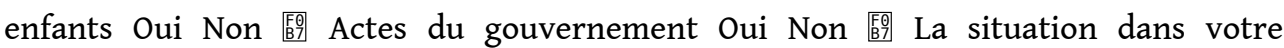
pénitencier Oui Non

31. Quelles sont les institutions auxquelles vous faites confiance? 臤 Les médias 緊 Les

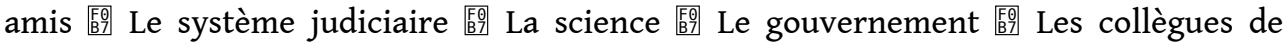

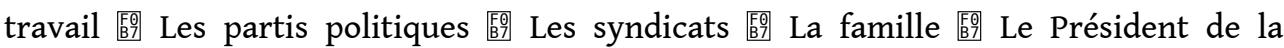

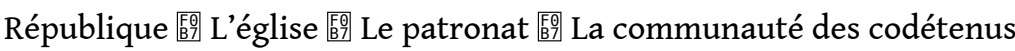

32. Serez-vous capable de vous adapter à la vie après la libération? 䀡 Oui, sans aucun

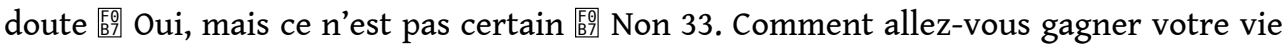

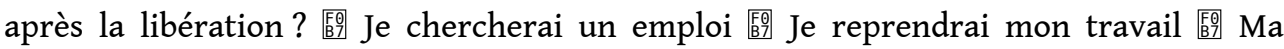

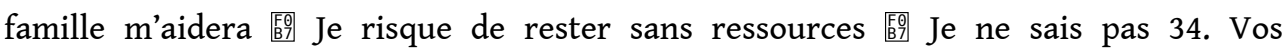
connaissances, vos qualités professionnelles vous permettront-elles de trouver facilement un travail ? 鹥 Oui 圆 Non 35. Quelles sont les normes qui sont indispensables

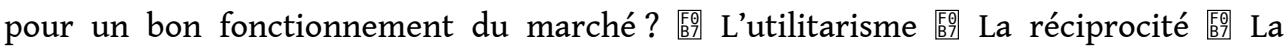

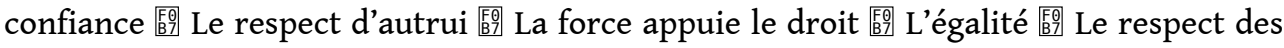

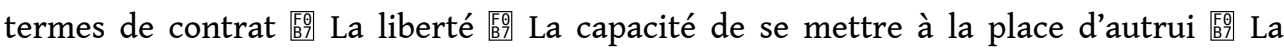
recherche d'un profit dans toute situation 㹂 La rationalité 36. Comment résoudriezvous un problème avec un fonctionnaire qui refuserait d'aider à vous réinsérer après la libération? 圆 Je m'adresserais à son supérieur hiérarchique 荡 J'utiliserais mes

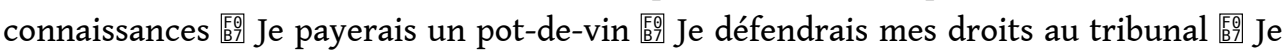
joindrais mes efforts à ceux qui se trouvent dans la même situation 㻣 Je ne sais pas

(mettre une croix dans la case correspondante) 29. Que prenez-vous en considération pour prendre une décision importante? 30 . Que pouvez-vous utiliser afin de résoudre

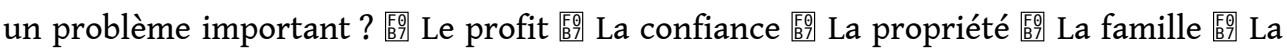

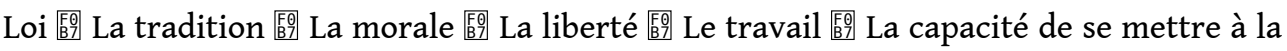

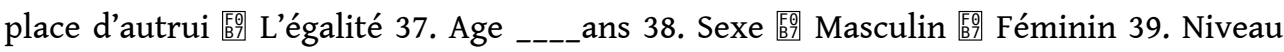

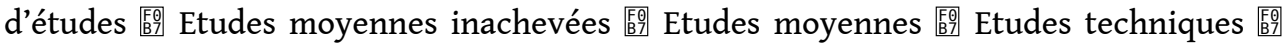

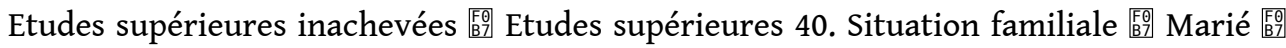



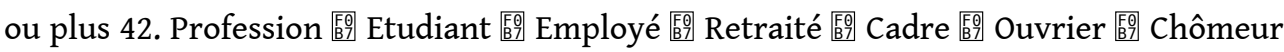

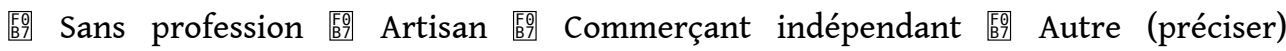
43. Durée de l'emprisonnement 荡 Inférieure à un an 落 Trois à

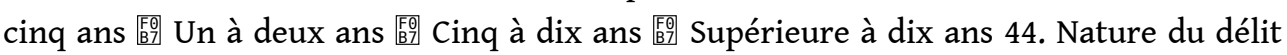
(d'après l'article du Code Pénal) 45. Etes-vous dans une cellule individuelle? 區 Oui 范 Non, il y a personnes

Retour a la première partie 


\section{NOTES}

1. Ibid.

2. Il s'agit des résultats d'une enquête pilote réalisée en 1996 sur un échantillon de 400 personnes dans 3 régions de la Fédération de Russie par l'Institut de politologie comparative de l'Académie des sciences, Moscou, sous la direction de S. Patrouchev.

3. Au cours de l'enquête réalisée en juin 1999 (cf. note n 46).

4. Levada Y., op. cit., p. 112 ; Rose R., Mishler W., « Mass Reaction to Regime Change in Eastern Europe : Polarization or Leaders and Laggards ? », British Journal of Political Science, Vol. 24, 1994, p. 208. Voir également nos enquêtes de 1996 et 1999.

5. Inglehart R., Culture Shift in Advanced Industrial Society, Princeton, Princeton University Press, 1990, p. 35.

6. Mansourov V. (dir.), Sovremennoe rossiiskoe obchtchestvo : perekhodny period (La société russe contemporaine : une période de transition), Moscou, Institout Sotsiologii RAN, 1999, pp. 17-18.

7. Levada Y., op. cit., p. 116.

8. Roché S., Le sentiment d'insécurité, Paris, PUF, 1993, p. 204.

9. Goriainov K., « Latentnaïa prestoupnost' v Rossii : rezoultaty issledovanii i mery borby » (La criminalité latente en Russie : résultats de recherches et mesures de lutte), in Latentnaïa prestoupnost' : poznanie, politika, strategia (La criminalité latente : connaissance, politique, stratégie), Moscou, VNII MVD RF, 1993, pp. 24-25.

10. Levada Y., op. cit., p. 266.

11. Seul 8,6 \% des personnes interrogées considèrent que ces réformes ont eu des effets positifs. Mansourov V., op. cit., p. 11.

12. Rossi J., Spravotchnik po GOULAGOU (Le guide du Goulag), Moscou, Prosvet, 1991, t. 1, p. 65.

13. Pour une analyse de la lutte contre les touneïadtsy, voir Favarel-Garrigues G., op. cit.

14. Voir, par exemple, Berelowitch A., Wieviorka M., Les Russes d'en bas. Enquête sur la Russie post-communiste, Paris, Seuil, 1996, pp. 61-63, p. 173, p. 246.

15. Girard R., op. cit., p. 375.

16. Mansourov V., op. cit., p. 16.

17. Ispravnikov V., Koulikov V., « Tenevaïa ekonomika v Rossii : inoï put' i tretia sila » (L'économie souterraine en Russie : autre voie et troisième force), Moscou, Rossiiskii ekonomitcheskii journal, 1997, p. 29 ; Kaufmann D., « Corruption in Transition Economies ", in Newman P. (ed.), New Palgrave Dictionary of Economics and the Law, Londres, Macmillan, 1998, Vol.1, p. 528.

18. Dolgova A., Diakov S. (dir.), op. cit., p. 212.

19. Dolgova A., Diakov S. (dir.), op. cit., pp.171-172.

20. Podlesskikh G., Terechonok A., Vory v zakone : brosok k vlasti (Les voleurs dans la loi : un bond vers le pouvoir), Moscou, Khoudojestvennaïa literatoura, 1995, p. 216.

21. Le pizzo « est fixé par le chef de famille en fonction des revenus supposés ou à venir du 'contribuable' » (Padovani M., Les dernières années de la Mafia, Paris, Gallimard, 1987, p. 62). 
22. Gourov A., « Organizovannaïa prestoupnost' i tenevaïa ekonomika » (La criminalité organisée et l'économie souterraine), in Tenevaïa ekonomika (L'économie souterraine), Moscou, Ekonomika, 1991, p. 124.

23. Il s'agit d'une proposition faite à la fin des années 1980. A l'époque, on appelait coopérateurs (kooperatory) les commerçants dont l'activité avait été légalisée par les arrêtés, puis la loi sur la coopération (1987-1988).

24. Rossiisko-Evropeiskii Tsentr Ekonomitcheskoï Politiki, Obzor ekonomiki Rossii. Osnovnye tendentsii razvitia. IV kvartal 1997 (Revue de l'économie russe. Tendances fondamentales de développement durant le quatrième trimestre 1997), Moscou, 1998, p. 242.

25. Voir l'article de Iakov Gilinski et Iakov Kostioukovski dans ce numéro.

26. Célèbre prison de Saint-Petersbourg.

27. L'interlocuteur $n^{\circ} 23$ nous a confié des observations qui vont dans le même sens.

28. A titre de référence, le salaire mensuel d'un ingénieur soviétique était à l'époque d'environ 120-130 roubles.

29. Comme en témoigne cet interlocuteur, certains fonctionnaires d'Etat font partie de la clientèle des « banquiers » criminels.

30. Nous marquons par un indice $(*)$ les entretiens effectués avec des entrepreneurs russes en novembre 1998 - juillet 1999.

31. Le terme de «toit » est employé dans la langue courante pour désigner la personne ou l'organisation, légale ou criminelle, qui protège les intérêts d'une entreprise ou d'un individu.

32. Doubov I., op. cit., p. 699.

33. Levada I., op. cit., p. 41.

34. Boettke P., "Credibility, Commitment and Soviet Economic Reform », in Lazear E.

(ed.), Economic Transition in Eastern Europe and Russia : Realities of Reform, Stanford, Hoover Institution Press, 1995, p. 253. C'est nous qui soulignons.

35. Padovani M., op. cit., Paris, Gallimard, 1987, p. 148.

36. Arestant est le nom donné à celui qui essaie de vivre selon les règles informelles en vigueur dans le monde carcéral sans pour autant trop se rapprocher des blatnye. Il s'agit donc d'une catégorie informelle d'individus, que l'on pourrait placer entre les moujiki et les blatnye.

INDEX

Mots-clés : criminalité, culture(s), mafias et crime organisé

Index géographique : Russie

Index chronologique : post-guerre froide 